\title{
ON SUBHARMONICITY OF THE CAPACITY OF THE SPECTRUM
}

\author{
ZBIGNIEW SLODKOWSKI
}

\begin{abstract}
It is shown that if $T_{\lambda}$ is an analytic operator valued function (or if $f, g$ belong to a uniform algebra $A$ ) then $n$th diameters and logarithmic capacity of $\sigma\left(T_{\lambda}\right)$ (or of the set $g\left(f^{-1}(\lambda)\right)$ ) are subharmonic functions of $\lambda$ (on a suitable domain).
\end{abstract}

1. Introduction. Let $B(X)$ denote the algebra of all bounded operators in a complex Banach space $X$ and let

$$
\lambda \rightarrow T_{\lambda}: G \rightarrow B(X)
$$

be an analytic operator function in a complex domain $G$. The behaviour of the multivalued function

$$
\lambda \rightarrow \sigma\left(T_{\lambda}\right)
$$

has attracted the attention of several authors:

Brown and Douglas in [4] asked if the spectral radius of (1) or the function (2) itself have any maximum properties. The answer was given by E. Vesentini [10], who proved in particular:

TheOREM 1 (VeSENTINI, 1968). If $\lambda \rightarrow T_{\lambda}: G \rightarrow B(X)$ is an analytic function then the function

$$
\lambda \rightarrow \log r\left(T_{\lambda}\right): G \rightarrow[-\infty,+\infty)
$$

is subharmonic.

This theorem has some interesting applications ([1], [8]), but its following corollary is even more useful [1, Théorème 1.2.2].

TheOREM 1' (AUPETIT, 1976). Under assumptions of Theorem 1 the function $\lambda \rightarrow \log \left(\operatorname{diam}\left(\sigma\left(T_{\lambda}\right)\right)\right): G \rightarrow[-\infty,+\infty)$ is subharmonic.

Analogous results hold also in uniform algebras, with spectrum replaced by the set

$$
g\left(f^{-1}(\lambda)\right)=\left\{g(m): m \in \mathscr{T}_{A}, f(m)=\lambda\right\}
$$

where $g, f$ belong to a uniform algebra $A$.

Received by the editors August 22, 1979 and, in revised form, November 20, 1979; presented to the Society, November 3, 1979.

AMS (MOS) subject classifications (1970). Primary 46J10; Secondary 47A55.

Key words and phrases. Spectrum, Shilov boundary, subharmonic functions, logarithmic capacity. 
TheOREM 2 (WERMER, 1975, AUPETIT, WeRMER, 1978). Let $A$ be a uniform algebra and let $X$ denote its Shilov boundary. Assume that $g, f \in A$ and $G=\sigma(f) \backslash f(X)$ is nonvoid. Then the following two functions

$$
\begin{aligned}
& \lambda \rightarrow \log \max \left|g\left(f^{-1}(\lambda)\right)\right|: G \rightarrow[-\infty,+\infty), \\
& \lambda \rightarrow \log \operatorname{diam}\left|g\left(f^{-1}(\lambda)\right)\right|: G \rightarrow[-\infty,+\infty),
\end{aligned}
$$

are subharmonic.

Since these results proved to be useful as well (cf. [1, pp. 99-106], [11]), B. Aupetit asked [1] if $\operatorname{diam}(\cdot)$ and $\max |(\cdot)|$ above could be replaced by some other functions of set argument, more specifically, whether the following functions were subharmonic

$$
\begin{gathered}
\lambda \rightarrow \log \delta_{k}\left(\sigma\left(T_{\lambda}\right)\right), \quad k=0,1,2, \ldots, \\
\lambda \rightarrow \log \operatorname{cap}\left(\sigma\left(T_{\lambda}\right)\right), \\
\lambda \rightarrow \log \delta_{k}\left(g\left(f^{-1}(\lambda)\right)\right), \quad k=0,1,2, \ldots, \\
\lambda \rightarrow \log \operatorname{cap}\left(g\left(f^{-1}(\lambda)\right)\right),
\end{gathered}
$$

where " $\delta_{k}$ " and "cap" stand for $k$ th diameter and logarithmic capacity of a compact plane set.

Special cases of these conjectures have been already known. Assuming that all $T_{\lambda}, \lambda \in G$, commute, B. Aupetit has shown subharmonicity of (3) and (4) (oral communication). D. Kumagai has proved that (5), (6) are subharmonic, if the algebra $A$ satisfies

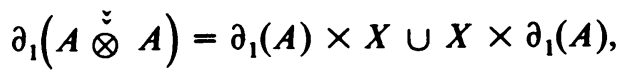

where $\partial_{1}(A)$ denotes some generalized Shilov boundary (cf. [3], [6]).

The aim of this note is to prove these conjectures without any restricting assumptions. We shall prove a little bit more.

Theorem 3. Let $\lambda \rightarrow T_{\lambda}: G \rightarrow B(X)$ be analytic and let $w$ be a complex polynomial in $k$ variables. Then the function

$$
\lambda \rightarrow \log \max \left|w\left(\sigma\left(T_{\lambda}\right) \times \cdots \times \sigma\left(T_{\lambda}\right)\right)\right|: G \rightarrow[-\infty,+\infty)
$$

is subharmonic.

Theorem 4. Let $A$ be a uniform algebra and $X$ its Shilov boundary. Assume that $f$, $g \in A$ and $G=\sigma(f) \backslash f(X)$ is nonvoid. Then for any complex polynomial $w\left(z_{1}, \ldots, z_{k}\right)$ the function

$$
\lambda \rightarrow \log \max \left|w\left(g\left(f^{-1}(\lambda)\right) \times \cdots \times g\left(f^{-1}(\lambda)\right)\right)\right|: G \rightarrow[-\infty,+\infty)
$$

is subharmonic.

The main result is a direct consequence of these theorems:

COROLlARY. Under assumptions of Theorems 1 and 2 functions (3)-(6) are subharmonic. 
Readers more interested in the background of discussed problems are referred to the book [1].

My grateful acknowledgement is due to Professor Bernard Aupetit for introducing me to the subject, attracting my attention to several interesting questions and many informative conversations.

I also would like to thank Professors M. D. Choi and M. Feder for valuable discussion of some unclear points.

2. Operator case. We first show how the Corollary follows from Theorems 3 and 4.

Proof. As it was noted by B. Aupetit, subharmonicity of (4), (6) follows from that of (3), (5), for $\delta_{k}(Z) \searrow \operatorname{cap}(Z)$ for any plane compact $Z$ and the limit of nonincreasing sequence of subharmonic functions is also subharmonic (for notions related to capacity or subharmonicity the reader is referred to [9] or to Appendix II in [1]). Let us recall that

$$
\log \delta_{k}(Z)=\left(\begin{array}{c}
k+1 \\
2
\end{array}\right)^{-1} \log \max \left|W_{k}(Z \times \cdots \times Z)\right|
$$

where

$$
W_{k}\left(z_{1}, \ldots, z_{k}\right)=\prod_{1<i<j<k}\left(z_{i}-z_{j}\right) .
$$

By Theorems 3, 4 the functions

$$
\lambda \rightarrow \log \max \left|W_{k}\left(\sigma\left(T_{\lambda}\right) \times \cdots \times \sigma\left(T_{\lambda}\right)\right)\right|
$$

and

$$
\lambda \rightarrow \log \max \left|W_{k}\left(g\left(f^{-1}(\lambda)\right) \times \cdots \times g\left(f^{-1}(\lambda)\right)\right)\right|
$$

are subharmonic. They are still so after dividing by the positive scalar $\left(\begin{array}{c}k+1 \\ 2\end{array}\right)$; applying (7) we get that (3), (5) are subharmonic. Q.E.D.

For the proof of Theorem 3 we need the following result of Dash and Schecter (it is actually only a special case of their Corollary 1 in [5]).

TheOREM 5 (DASH, Schecter, 1970). Let $X$ be a complex Banach space and let $Y=X \hat{\otimes} \cdots \hat{\otimes} X$ denote complete $k$-fold projective tensor product. Let $T \in B(X)$ and $T_{i}=I \otimes \cdots \otimes T \otimes \cdots \otimes I, i=1, \ldots, k$. Then operators $T_{1}, \ldots, T_{k}$ commute and for any complex polynomial $w\left(z_{1}, \ldots, z_{k}\right)$ we have

$$
\sigma\left(w\left(T_{1}, \ldots, T_{k}\right)\right)=w(\sigma(T) \times \cdots \times \sigma(T)) .
$$

Proof of Theorem 3. Denote by $T_{i}(\lambda)$ the bounded operator in $Y$, determined uniquely by $I \otimes \cdots \otimes T_{\lambda} \otimes \cdots \otimes I, i=1, \ldots, k, \lambda \in G$. Put

$$
W_{\lambda}=w\left(T_{1}(\lambda), \ldots, T_{k}(\lambda)\right) \text {. }
$$

Clearly $W_{\lambda}$ is an analytic function of $\lambda$. By the Vesentini theorem $\lambda \rightarrow \log r\left(W_{\lambda}\right)$ is subharmonic. On the other hand

$$
\begin{aligned}
r\left(W_{\lambda}\right) & =\max \left|\sigma\left(W_{\lambda}\right)\right|=\max \left|\sigma\left(w\left(T_{1}(\lambda), \ldots, T_{k}(\lambda)\right)\right)\right| \\
& =\max \left|w\left(\sigma\left(T_{\lambda}\right) \times \cdots \times \sigma\left(T_{\lambda}\right)\right)\right|
\end{aligned}
$$


(by Theorem 5). Thus

$$
\lambda \rightarrow \log \max \left|w\left(\sigma\left(T_{\lambda}\right) \times \cdots \times \sigma\left(T_{\lambda}\right)\right)\right|
$$

is subharmonic. Q.E.D.

3. Uniform algebras. The following folklore lemma is proved here for the convenience of the reader.

Lemma. $A$ and $X$ as in Theorem 4. Assume that $f \in A$ and $\lambda_{0} \notin f(X)$. Then there is $\varepsilon>0$ such that for every $m_{0} \in A^{*}$ with $m_{0} \perp\left(f-\lambda_{0}\right) A$ there is an analytic vector function

$$
\lambda \rightarrow m(\lambda): D\left(\lambda_{0}, \varepsilon\right) \rightarrow A^{*}
$$

such that

$$
m(\lambda) \perp(f-\lambda) A, \quad m\left(\lambda_{0}\right)=m_{0}
$$

and

$$
\|m(\lambda)\|<2\left\|m_{0}\right\|
$$

for $\lambda \in D\left(\lambda_{0}, \varepsilon\right)$.

Proof. Without loss of generality we can assume that $\lambda_{0}=0$. By [7, Corollary 3.3.7] there is $\Delta>0$ such that

$$
\|f g\|>\|g\| / \Delta \text { for all } g \in A \text {. }
$$

In particular, $f A$ is closed. Consider action of $A$ on $A^{*}$ defined by

$$
(f \cdot m)(g):=m(f g), \quad f, g \in A, m \in A^{*} .
$$

With (10) we have

for any $m \in A^{*}$ there is $m^{\prime} \in A^{*}$ such that $m=f \cdot m^{\prime}$ and $\left\|m^{\prime}\right\|<\Delta\|m\|$.

Indeed. Consider linear functional

$$
f g \rightarrow m(g): f A \rightarrow C .
$$

Its norm is not greater than $\|m\| \Delta$ :

$$
|m(g)| \leqslant\|m\| \cdot\|g\|<(\|m\| \Delta) \cdot\|f g\|,
$$

so it can be extended to a functional $m^{\prime}$ on $A$ with the same norm (Hahn-Banach theorem). Such $m^{\prime}$ satisfies (*). Applying (*) inductively (starting from given $m_{0}$ ) we find $m_{n} \in A^{*}, n=1,2, \ldots$, such that

$$
m_{n-1}=f \cdot m_{n}, \quad\left\|m_{n}\right\|<\Delta^{n}\left\|m_{0}\right\|, \quad n=1,2, \ldots
$$

Put $\varepsilon=(2 \Delta)^{-1}$. In $D(0, \varepsilon)$ the series

$$
m(\lambda)=\sum_{n=0}^{\infty} m_{n} \lambda^{n}
$$

is absolutely convergent to an analytic vector-function; moreover $\|m(\lambda)\|<2\left\|m_{0}\right\|$. Using (11) we compute that

$$
(f-\lambda) m(\lambda)=f m_{0}+\sum_{n=1}^{\infty}\left(f m_{n}-m_{n-1}\right) \lambda^{n}=f m_{0} .
$$


By assumptions $m_{0}$ annihilates $f A$, i.e. $f m_{0}=0$. Therefore

$$
(f-\lambda) m(\lambda) \equiv 0, \quad \lambda \in \Delta\left(\lambda_{0}, \varepsilon\right) \text {. Q.E.D. }
$$

We shall prove Theorem 4 using the following criterion for subharmonicity:

Proposition. Let $G \subset C$ and $\phi: G \rightarrow[-\infty,+\infty)$ be upper semicontinuous. Assume that for every $\lambda_{0} \in G$ there exist $\varepsilon>0$ and function $\psi$ defined in $D\left(\lambda_{0}, \varepsilon\right)$ such that

(i) $\psi\left(\lambda_{0}\right)=\phi\left(\lambda_{0}\right)$,

(ii) $\psi(\lambda) \leqslant \phi(\lambda), \lambda \in D\left(\lambda_{0}, \varepsilon\right)$,

(iii) $\psi$ is uniformly bounded from the above Baire class and satisfies the mean-value inequality, i.e.

$$
\psi\left(\lambda_{0}\right) \leqslant \frac{1}{2 \pi} \int_{0}^{2 \pi} \psi\left(\lambda_{0}+r e^{i \theta}\right) d \theta, \quad 0<r<\varepsilon .
$$

Then $\phi$ is subharmonic in $G$.

This proposition is an immediate consequence of a classical test for subharmonicity, see $[13, \S 9,4$, p. 58].

Proof of Theorem 4. We have to show that the function

$$
\phi(\lambda)=\log \max \left\{\left|w\left(z_{1}, \ldots, z_{k}\right)\right|: z_{i} \in g\left(f^{-1}(\lambda)\right), i=1, \ldots, k\right\},
$$

is subharmonic in $G=\sigma(f) \backslash f(X)$. Observe first that $\phi$ is upper semicontinuous, for $\lambda \rightarrow g\left(f^{-1}(\lambda)\right)$ is an upper semicontinuous set-valued function (we omit the details).

By the proposition it remains to show that for an arbitrary $\lambda_{0} \in G$ we can find a positive $\varepsilon$ and a function $\psi$ defined in $D\left(\lambda_{0}, \varepsilon\right)$ such that conditions (i)-(iii) are satisfied.

Fix $\lambda_{0} \in G$. To define $\psi$ observe first that the maximum in (12) is actually attained, therefore we can choose multiplicative-linear functions $m_{1}, \ldots, m_{k} \in$ $f^{-1}\left(\lambda_{0}\right)$ such that

$$
\phi\left(\lambda_{0}\right)=\log \left|w\left(g\left(m_{1}\right), \ldots, g\left(m_{k}\right)\right)\right| .
$$

Since $m_{i}(f)=\lambda_{0}$, clearly $m_{i}$ annihilate $\left(f-\lambda_{0}\right) A$ and we can apply the lemma to "initial values" $m_{0}=m_{i}, i=1, \ldots, k$. We obtain $\varepsilon>0$ and $k$ analytic vectorfunctions

$$
\lambda \rightarrow m_{i}(\lambda): D\left(\lambda_{0}, \varepsilon\right) \rightarrow A^{*}
$$

such that $m_{i}\left(\lambda_{0}\right)=m_{i}$ and conditions (8), (9) are satisfied.

Let $B=A \hat{\otimes} A \hat{\otimes} \cdots \hat{\otimes} A$ be the $k$-fold tensor product completed with respect to projective norm. With natural multiplication $B$ becomes a commutative Banach algebra with unit. Put $g_{i}=e \otimes \cdots \otimes g \otimes \cdots \otimes e$. We can consider $\tilde{g}:=w\left(g_{1}, \ldots, g_{k}\right)$ and $\tilde{g}^{n}$. Finally we define

$$
\psi(\lambda):=\lim \sup _{n}\left(\frac{1}{n} \log \left|\left\langle\tilde{m}(\lambda), \tilde{g}^{n}\right\rangle\right|\right), \quad \lambda \in D\left(\lambda_{0}, \varepsilon\right),
$$

where $\tilde{m}(\lambda)$ is the unique element of $B^{*}$ determined by $m_{1}(\lambda) \otimes \cdots \otimes m_{k}(\lambda)$.

To end the proof we have to check that conditions (i)-(iii) are satisfied. 
Condition (i). Since $\tilde{m}\left(\lambda_{0}\right)=m_{1} \otimes \cdots \otimes m_{k}$ is a multiplicative-linear functional on $B$, therefore

$$
\begin{aligned}
w\left(\left\langle m_{1}, g\right\rangle, \ldots,\left\langle m_{k}, g\right\rangle\right)^{n} & =w\left(\left\langle\tilde{m}\left(\lambda_{0}\right), g_{1}\right\rangle, \ldots,\left\langle\tilde{m}\left(\lambda_{0}\right), g_{k}\right\rangle\right)^{n} \\
& =\left\langle\tilde{m}\left(\lambda_{0}\right), w\left(g_{1}, \ldots, g_{k}\right)\right\rangle^{n} \\
& =\left\langle\tilde{m}\left(\lambda_{0}\right), w\left(g_{1}, \ldots, g_{k}\right)^{n}\right\rangle=\left\langle\tilde{m}\left(\lambda_{0}\right), \tilde{g}^{n}\right\rangle .
\end{aligned}
$$

Thus

$$
\lim \sup _{n} \frac{1}{n} \log \left|\left\langle\tilde{m}\left(\lambda_{0}\right), \tilde{g}^{n}\right\rangle\right|=\log \left|w\left(m_{1}(g), \ldots, m_{k}(g)\right)\right|,
$$

which proves Condition (i).

Condition (ii). Fix $\lambda \in D\left(\lambda_{0}, \varepsilon\right)$ and denote by $J_{\lambda}$ the closed ideal in $B$ generated by the following $k$ elements:

$$
e \otimes e \otimes \cdots \otimes(f-\lambda e) \otimes \cdots \otimes e, \quad i=1, \ldots, k .
$$

Since all $m_{i}(\lambda)$ satisfy conditions (8), (9) of the lemma, $\tilde{m}(\lambda)$ annihilates $J_{\lambda}$ and $\|\tilde{m}(\lambda)\|<2^{k}\left\|m_{1}\right\| \cdots\left\|m_{k}\right\|=2^{k}$. Therefore

$$
\left|\left\langle\tilde{m}(\lambda), \tilde{g}^{n}\right\rangle\right| \leqslant\|\tilde{m}(\lambda)\| \cdot\left\|\tilde{g}^{n}+J_{\lambda}\right\|<2^{k}\left\|\tilde{g}^{n}+J_{\lambda}\right\|,
$$

and

$$
\lim \sup _{n}\left(\frac{1}{n} \log \left|\left\langle\tilde{m}(\lambda), \tilde{g}^{n}\right\rangle\right|\right)<\log \left(\lim \sup _{n} \sqrt[n]{\left\|\tilde{g}^{n}+J_{\lambda}\right\|}\right),
$$

where $\left\|\tilde{g}^{n}+J_{\lambda}\right\|$ denotes the norm of the coset $\left[\tilde{g}^{n}\right]$ in the quotient algebra $B / J_{\lambda}$. By the spectral radius formula the last inequality reads

$$
\psi(\lambda)<\log r_{\lambda}([\tilde{g}])
$$

where $r_{\lambda}(\cdot)$ denotes spectral radius with respect to $B / J_{\lambda}$.

The Gelfand space of $B / J_{\lambda}$ can be identified with the annihilator of $J_{\lambda}$ in $\mathfrak{R}_{B}$ (cf. [12, Theorem 11.4]). Therefore there are $m_{1}^{\prime}, \ldots, m_{k}^{\prime} \in \mathfrak{T}_{A}$ such that $\tilde{m}=m_{1}^{\prime}$ $\otimes \cdots \otimes m_{k}^{\prime}$ annihilates $J_{\lambda}$ and

$$
r_{\lambda}([\tilde{\boldsymbol{g}}])=|\langle\tilde{m}, \tilde{\boldsymbol{g}}\rangle|
$$

In particular it holds

$$
\begin{aligned}
0 & =\langle\tilde{m}, e \otimes \cdots \otimes(f-\lambda e) \otimes \cdots \otimes e\rangle \\
& =\left\langle m_{i}^{\prime}, f-\lambda e\right\rangle \cdot \prod_{j \neq i}\left\langle m_{j}^{\prime}, e\right\rangle=f\left(m_{i}^{\prime}\right)-\lambda,
\end{aligned}
$$

hence $m_{i}^{\prime} \in f^{-1}(\lambda), i=1, \ldots, k$. Similarly as in (14) we can get

$$
\langle\tilde{m}, \tilde{g}\rangle=w\left(g\left(m_{1}^{\prime}\right), \ldots, g\left(m_{k}^{\prime}\right)\right) \text {, }
$$

and by definition of $\phi$

$$
\phi(\lambda) \geqslant \log \left|w\left(g\left(m_{1}^{\prime}\right), \ldots, g\left(m_{k}^{\prime}\right)\right)\right|=\log |\langle\tilde{m}, \tilde{g}\rangle| .
$$

This relation together with (17) and (16) proves Condition (ii).

Condition (iii). From (15) it follows in particular that

$$
\left\langle\tilde{m}(\lambda), \tilde{g}^{n}\right\rangle \mid<\left(2^{k}\|\tilde{g}\|\right)^{n}, \quad \lambda \in D\left(\lambda_{0}, \varepsilon\right), n=1,2, \ldots,
$$


therefore functions

$$
\lambda \rightarrow \frac{1}{n} \log \left|\left\langle\tilde{m}(\lambda), \tilde{g}^{n}\right\rangle\right|: D\left(\lambda_{0}, \varepsilon\right) \rightarrow[-\infty,+\infty), \quad n=1,2, \ldots,
$$

are uniformly bounded from above. We omit the simple argument which shows that the functions $\lambda \rightarrow\left\langle\tilde{m}(\lambda), \tilde{g}^{n}\right\rangle$ are analytic and conclude that functions (18) satisfy mean value inequality. This and uniform boundedness imply that $\psi(\lambda)$ satisfies the mean-value inequality. Q.E.D.

ACKNOWledgement. This research was done in Spring 1979 while the author was visiting in the Department of Mathematics, University of Toronto. The author is most grateful to the department and especially to Professor Peter Rosenthal for their friendly hospitality.

\section{REFERENCES}

1. B. Aupetit, Propriétés spectrales des algèbres de Banach, Lecture Notes in Math., vol. 735, Springer-Verlag, Berlin and New York, 1979.

2. Z. Slodkowski, Analytic set-valued functions and spectra (in preparation)

3. R. Basener, A generalized Shilov boundary and analytic structure, Proc. Amer. Math. Soc. 47 (1975), 98-104.

4. A. Brown and R. G. Douglas, On maximum theorems for analytic operator functions, Acta Sci. Math. Szeged 26 (1966), 325-327.

5. A. T. Dash and M. Schechter, Tensor products and joint spectra, Israel J. Math. 8 (1970), $191-193$.

6. D. Kumagai, Subharmonic functions and uniform algebras, Proc. Amer. Math. Soc. 78 (1980), 23-29.

7. C. E. Rickart, General theory of Banach algebras, Van Nostrand, Princeton, N.J., 1960.

8. Z. Slodkowski, W. Wojtynski and J. Zemanek, A note on quasi-nilpotent elements of a Banach algebra, Bull. Acad. Polon. Sci. Ser. Sci. Math. Astronom. Phys. 25 (1977), 131-134.

9. M. Tsuji, Potential theory in modern function theory, Maruzen, Tokyo, 1959.

10. E. Vesentini, On the subharmonicity of the spectral radius, Boll. Un. Mat. Ital. 4 (1968), 427-429.

11. J. Wermer, Subharmonicity and hulls, Pacific J. Math. 58 (1975), 283-290.

12. W. Żelazko, Banach algebras, Elsevier, Amsterdam, 1973.

13. V. S. Vladimirov, Methods of the theory of functions of many complex variables, The M.I.T. Press, Cambridge, Mass., 1966.

Department of Mathematics, University of Toronto, Toronto, Ontario, Canada M5S 1A1

Current address: Institute of Mathematics, Polish Academy of Sciences, Sniadeckich 8, Warsaw, Poland 